\title{
A Pair of Identified Interneurons in Aplysia that Are Involved in Multiple Behaviors Are Necessary and Sufficient for the Arterial- Shortening Component of a Local Withdrawal Reflex
}

\author{
Yuanpei Xin, ${ }^{1}$ Klaudiusz R. Weiss, ${ }^{2}$ and Irving Kupfermann ${ }^{1}$ \\ ${ }^{1}$ Center for Neurobiology and Behavior, College of Physicians and Surgeons, Columbia University, New York, New York \\ 10032, and 2Department of Physiology and Biophysics, Mt. Sinai Medical Center, New York, New York 10029
}

\begin{abstract}
A bilateral pair of cerebral interneurons, called CC5, contribute to the generation of a number of different behaviors involving head movements. Each cell sends its axon to the ipsilateral and contralateral pedal and pleural ganglia. A weak tactile stimulus to the head excites the ipsilateral CC5; a strong stimulus excites both the ipsilateral and contralateral cells. Firing of CC5 produces powerful shortening of the ipsilateral pedal artery (PA) by means of monosynaptic excitation of the pedal artery shortener (PAS) neuron, the single motor neuron for the artery. A weak touch to a tentacle excites the ipsilateral PAS and evokes a local withdrawal response accompanied by shortening of the ipsilateral PA. In vivo recording of the pedal artery nerve (PAn) showed that PAS was activated bilaterally during defensive head withdrawal elicited by a strong stimulus and was activated unilaterally by a weak stimulus. The responses were eliminated
\end{abstract}

by cutting the ipsilateral cerebral-pleural connective (C-PLC). Electrical stimulation of the cerebral-pleural connective provided evidence that all of the excitatory input to PAS via this connective is provided by CC5. A variety of experimental results indicates that during a local withdrawal reflex of the tentacle, CC5 is necessary and sufficient for the unilateral PA-shortening component of the response and therefore functions as a command neuron for a component of the behavior. The data suggest that during defensive head withdrawal, the two CC5 neurons may act conjointly as a two-neuron command system that is necessary and sufficient for the bilateral arterial-shortening component of the behavior.

Key words: command; withdrawal reflex; Aplysia; mechanosensory; feeding; head turning
A major task in neuroscience is to understand the functioning of the nervous system in terms of the activity of individual neurons or aggregates of neurons. Studies of invertebrates and vertebrates have shown that certain interneurons appear to be extraordinarily focused in their function (Kupfermann and Weiss, 1978; Nolen and Hoy, 1984; Arshavsky et al., 1988; Didomenico and Eaton, 1988; McCrohan and Kyriakides, 1989; Delaney and Gelperin, 1990; Young and Yamane, 1992; Celebrini and Newsome, 1994; Frost and Katz, 1996) and that there are a small number of cells that appear to act as so-called command neurons, i.e., individual cells that meet the criteria of necessity and sufficiency for a particular behavior (Kupfermann and Weiss, 1986). One problem with the concept of command neurons is that there are good theoretical reasons (Getting, 1989; Churchland and Sejnowski, 1992) as well as growing empirical evidence that neurons can be involved in more than one behavior, and that their role in different behaviors may not be invariant (Hooper and Moulins, 1989; Lockery and Kristan, 1990; Fetz, 1992; Meyrand et al., 1994; Wu et al., 1994). In the nervous system of vertebrates and many higher invertebrates, the evidence for multifunctionality of interneurons, however, is indirect, and the neural circuitry and behavior are so

Received Feb. 16, 1996; revised April 24, 1996; accepted April 26, 1996.

This work was supported in part by National Institutes of Health Grants MH36730, GM32099, and MH35564. We thank S. C. Rosen for comments on this paper.

Correspondence should be addressed to Irving Kupfermann, Center for Neurobiology and Behavior, College of Physicians and Surgeons, Columbia University, 722 West 168th Street, Research Annex, New York, NY 10032.

Copyright (C) 1996 Society for Neuroscience $0270-6474 / 96 / 164518-11 \$ 05.00 / 0$ complex that it is not possible to understand unambiguously how the activity of the neurons relates to specific behaviors.

Head movement in Aplysia provides a good model system for exploring issues concerned with the functional role of neurons in multiple behaviors. Head movements occur in a variety of behaviors (Leonard and Lukowiak, 1986) including defensive withdrawal (Walters, 1986; Teyke et al., 1989), locomotion and searching (Teyke et al., 1992; Kuenzi and Carew, 1994), feeding (Preston and Lee, 1973; Kupfermann, 1974a; Teyke et al., 1990b), and reproductive behavior (Arch and Smock, 1977; Susswein et al., 1984; Ferguson et al., 1989; Bernheim and Mayeri, 1995). Each of these behaviors involves a head movement component that occurs together with other behavioral components specific to the particular response. For example, feeding involves a coordination of relatively nonstereotyped appetitive behaviors involving head movements and more stereotyped regular rhythmic consummatory responses, such as biting and swallowing, that are executed by the buccal mass.

There are at least three distinct variants of head movements in Aplysia: (1) head extension and retraction (occurring during locomotion and defensive responses), (2) nondirected head waving (occurring during free foraging), and (3) directed head turning (occurring in response to a unilateral food stimulus and to noxious stimuli, and during reproductive behaviors). Because directed head turning is a unilateral response to a unilateral stimulus, in the present study, we attempted to find candidate neurons for head turning by identifying cells that have asymmetrical inputs and outputs. We present the findings on one such cell, an interneuron called CC5. Evidence is provided that CC5 neurons are involved in the control of a component of two types of move- 
ments: a local, tentacle withdrawal, and a defensive head withdrawal. Both of these responses involve contractions of muscles of the neck and head and, in addition, include a shortening response of one or both pedal arteries. Either individual CC5 cells or the left-right pair of cells may be necessary and sufficient for the arterial-shortening component of these responses. During withdrawal responses, CC5 appears to provide the only excitation of the pedal artery shortener neuron (PAS), a motor neuron that provides the only source of excitation of the PA (Skelton and Koester, 1992). Elsewhere, we provide evidence that CC5 plays various roles in a number of other behaviors (Xin et al., 1996).

\section{MATERIALS AND METHODS}

Subjects. These experiments were done on $\sim 200$ wild-type Aplysia californica, weighing 250-350 gm (Marinus, Long Beach, CA). The animals were maintained at $14-16^{\circ} \mathrm{C}$ in holding tanks containing aerated, filtered artificial seawater (ASW) for 3-6 d before being used for experiments.

Preparations. Three types of preparations were used: an isolated ganglia preparation, a semi-intact (isolated head) preparation, and a reduced preparation. Before dissection, the animal was immobilized by an injection of an isotonic magnesium chloride solution of $\sim 25 \%$ of body volume.

The isolated ganglia preparation included all the head ganglia (buccal, cerebral, pedal, and pleural). In some experiments, as specified, we also included the abdominal ganglion or pedal arteries. All ganglia, except the buccal, were pinned dorsal side up to a clear Sylgard silicone elastomer floor of a recording chamber containing fresh ASW. The buccal ganglion was pinned rostral surface up. For the preparations of head ganglia with the pedal arteries attached, the arteries were carefully dissected bilaterally. Special care was taken to protect the pair of very fine pedal nerves that innervate the arteries and control the movement of the arterial longitudinal muscles (Skelton and Koester, 1992).

The isolated head preparation consisted of the head ganglia, mouth, lips, and anterior tentacles with all the associated nerves from the cerebral ganglia, i.e., upper labial nerve, lower labial nerve, and anterior tentacle nerve. The cephalic artery, which supplies the mouth, lip, and anterior tentacle regions of the animal (Skelton and Koester, 1992) also was preserved for subsequent perfusion. The preparations were set in a clear Lucite recording chamber consisting of two compartments containing ASW. The head ganglia were pinned out in one compartment in the manner described above for the isolated ganglia preparation. The mouth, lip, tentacles, and artery were set in the second compartment, which was deeper than the first chamber, so that the tissue could be immersed completely in the ASW. The partition between the two compartments contained fine grooves that allowed the peripheral nerves to pass through. The grooves then were filled with Vaseline to maintain a watertight seal between the two compartments. The cephalic artery was cannulated, and fresh ASW was pumped into the artery at a rate of about $0.5 \mathrm{ml}$ per minute to perfuse the tissue and simulate a hydroskeleton. A suction tube for the outflow was set in the compartment to control the fluid level.

The reduced preparation consisted of the head ganglia and half of the anterior body wall with the pedal artery (PA) attached (Nagahama et al., 1993), and with lengths of pedal nerves 4, 5, and 7 (P4, P5, P7), and pleural nerve 1 (PL1). The preparation was set in a chamber containing two compartments. The head ganglia were pinned in one compartment, dorsal side up. The body wall was pinned in the second compartment with the inner layer up so that it was possible to visualize the foot and neck muscle and the regions where each nerve terminated. The PA was cannulated to allow the perfusion of fresh ASW, using the same system described for the semi-intact preparation. The viability of the muscle was improved greatly by perfusion of the tissue, permitting the recording of the relatively small contractions of the neck muscles (Nagahama et al., 1993). Each reported result is based on observations on at least 3 preparations and in many cases, 5-10 preparations.

Stimuli. Mechanical stimuli were provided by the tip of a heat-sealed glass Pasteur pipette or by series of von Frey hairs. The von Frey hairs consisted of polyethylene filaments of different diameters that exerted a range of forces from 0.1 to $1.0 \mathrm{gm}$. The finest filament barely dimpled the skin of the animal, whereas the thickest filament was capable of damaging the skin in some instances. Combined chemo-mechano stimuli consisted of pieces of moistened dried-seaweed (Laver, Vega Trading Company, NY) that were applied to the lips or tentacles with a fine, blunt plastic forceps. The chemical stimulus consisted of a seaweed extract solution (Susswein et al., 1978) that was applied $1 \mathrm{~cm}$ from the animal by means of a $1 \mathrm{ml}$ syringe. The extract was ejected into the ASW at $\sim 2 \mathrm{ml} / \mathrm{min}$, a rate slow enough to be unlikely to produce any substantial tactile input, and application of ASW without extract was used as a control.

Electrophysiology. All in vitro experiments were performed at room temperature $\left(19-21^{\circ} \mathrm{C}\right)$. For the intracellular recording and stimulation, neurons were impaled with double-barreled microelectrodes that were made of thin-walled glass (World Precision Instruments, Sarasota, FL) and contained $2 \mathrm{~m}$ potassium acetate. The electrodes were flow beveled so that their impedances ranged from 10 to $15 \mathrm{~m} \Omega$. In some experiments, one electrode was filled with 3\% 5(6)-carboxyfluorescein dye (Rao et al., 1986 ) or with $2 \%$ Lucifer yellow (Stewart, 1978). To test for monosynapticity of connections, the threshold for action potential generation was raised by bathing the ganglia in a high-divalent cation solution. Unless otherwise specified, this solution contained $30 \mathrm{mM} \mathrm{Ca}^{2+}(3 \times$ normal $)$ and $150 \mathrm{mM} \mathrm{Mg}^{2+}(3 \times$ normal $)$.

For the extracellular recording or stimulation, various nerves and connectives were used including pleural and pedal nerves (PL1, P4, P5, P7), buccal nerves (B2) (Gardner, 1971), cerebral-buccal connective (C-BC), cerebral-pedal connective (C-PC), cerebral-pleural connective (C-PLC), and pleural-abdominal connective (PL-AbC). The cut ends of the nerves or connectives were drawn into small-diameter polyethylene suction electrodes. Nerve recordings were made with AC amplifiers (A-M Systems), and electrical stimulation of the nerves was provided by a Grass 88 stimulator.

For monitoring the contractions of muscles or blood vessels, an isotonic displacement transducer (Harvard Bioscience) was used. The transducer was set to record contractions along either transverse or longitudinal directions of the neck muscles (Nagahama et al., 1993) or was attached to the distal end of the PA (Skelton and Koester, 1992) so that it recorded changes in length of the artery.

Morphology. To determine the size, shape, and destination of processes of identified neurons, cells were filled with 3\% 5(6)-carboxyfluorescein dye. Successful intracellular labeling was achieved by iontophoretic injection of the dye for $15-60 \mathrm{~min}$, followed by a $48 \mathrm{hr}$ incubation at $4^{\circ} \mathrm{C}$ to allow the dye to fill the processes fully. To reduce active transport of the dye from the cells during incubation, probenecid (Steinberg et al., 1987) was added to the bathing solution (10 mm final concentration). As reported previously, the presence of probenecid greatly improved the dye fills, particularly for small processes far from the cell body (Rosen et al., 1991). After incubation, the living ganglia were cleared in $50 \%$ glycerol in ASW, the fluorescence was visualized with a Leitz fluorescence microscope, and the labeled cell body with its processes was photographed. In some preparations, the cells were filled with Lucifer yellow, fixed in paraformaldehyde, and viewed after dehydration and clearing. Finally, cells in the cerebral ganglion that project to the pedal and pleural ganglia were visualized by "back fills" of combinations of pairs of left and right pedal and pleural connectives, using $1 \mathrm{M}$ nickel chloride for one connective and and $1 \mathrm{~m}$ cobalt chloride for the contralateral connective. The cerebral ganglion then was developed with a saturated alcohol/rubeanic acid solution (dithio-oxamide, Sigma, St. Louis), fixed in formaldehyde, dehydrated, and cleared in methyl salicylate (Quicke and Brace, 1979).

In vivo recording of the pedal artery nerve $(P A n)$ before and after lesion of the $C$-PLC. To obtain information about possible functional roles of CC5, lesion experiments were performed on 15 animals. Animals were immobilized as mentioned above. All instruments and containers were sterilized by exposing them overnight to UV light. The surgical incision was started $5 \mathrm{~mm}$ lateral to a rhinophore and ran caudally for $5 \mathrm{~mm}$. The activity of the PAS neuron, which innervates the PA, was monitored by means of chronic in vivo recordings from the PAn, which contains the axon of the PAS neuron. The chronic recording techniques have been described previously (Alevizos et al., 1991; Skelton and Koester, 1992). In brief, to stabilize the small silicone polymer electrode, it was attached to the outside of a short length $(3 \mathrm{~mm})$ of polyethylene tubing. The tube (and attached electrode) was held in place by anchoring it to a portion of the arterial tree close to the nerve from which the recording was to be made. To anchor the tube, the artery was severed, and the flanged tips of the tube were inserted into the cut ends of the vessels. The vessel was tied around the ends of the polyethylene tube with silk sutures (Ethicon 6-0), leaving the electrode exposed between the cut ends of the vessel and permitting unimpeded blood flow. The intact nerve was placed on the groove on the electrode and was insulated from the hemocoel by pinning a latex membrane over the nerve. To record from the right PAn (RPAn), the tube-mounted electrode was inserted into the adjacent portion of the rostral anterior aorta. To record from the left PAn (LPAn), the tubemounted electrode was inserted into the proximal end of the left PA. 
After mounting of the electrode, the incision was closed with silk sutures (Ethicon 6-0), and the animal was returned to its home cage. Animals typically recovered from the operation after 1 or $2 \mathrm{~d}$. Animals were considered to have recovered from surgery when they responded to a food stimulus by assuming an upright feeding posture and showing bite responses when food was touched to the perioral zone. Movements of the animal and activity of the PAS were recorded simultaneously by using two video cameras, one of which was focused on the animal and the other focused on the paper of a Brush pen recorder. The two images were combined (Panasonic, Digital AV Mixer, WJ-AVE5) before recording on tape. After recording, the animals were subjected to a second operation to eliminate the direct input of CC5 to the PAS. This was accomplished by severing the C-PLC on the side from which the PAS was recorded, because CC5 sends its sole axon to the pedal ganglion via the ipsilateral C-PLC. Animals then were permitted to recover, and their behavior and PAS activity were recorded a second time.

\section{RESULTS}

As a first approach toward investigating possible neurons involved in head turning and other aspects of the appetitive phases of feeding, we attempted to locate cerebral interneurons that project to the pleural or pedal ganglia. The cerebral ganglion is in a position in which it can receive afferent information from the lips and tentacles and exert control over the head, neck, and foot, either directly by projections to these structures or indirectly by means of cerebral interneurons that project to neurons in the pedal and pleural ganglia. Indeed, there is evidence that the cerebral ganglion contains several feeding-related command-like neurons (Teyke et al., 1990a; Rosen et al., 1991) and locomotion command-like neurons (Fredman and Jahan-Parwar, 1983). Electrophysiological and anatomical data suggest that the C-PC or C-PLC contains axons of neurons that project from the cerebral ganglion to the pedal-pleural ganglia (Jahan-Parwar and Fredman, 1976; Teyke et al., 1989), and it is known that bilateral transection of the C-PC/C-PLC eliminates head-turning responses (Kupfermann, 1974b; Xin and Kupfermann, 1995).

Our backfills revealed the positions of cell bodies that send axons into the C-PC or C-PLC (Fig. 1). Backfill of the C-PC revealed cells positioned throughout the major cell cluster groups (Jahan-Parwar and Fredman, 1976) of the cerebral ganglion (Fig. $1 A$ ). In some preparations, one or two filled cells could be found in the ipsilateral E cluster. Intracellular dye fills of cells in the $\mathrm{E}$ cluster revealed a reproducibly identified cell that sends its axon into the ipsilateral pedal connective. Backfill of the C-PL (Fig. 1B) filled cells located primarily in the A, D, and C clusters, and no cells were found in the E cluster.

In an initial intracellular survey of cells that send an axon to the pedal or pleural ganglia, we found that the C cluster (Fig. 1, circled cells) contains a number of neurons that appear to have largely unilateral inputs and outputs, making them good candidates for participation in strongly lateralized responses such as head turning. A general survey revealed that the $\mathrm{C}$ cluster can be divided approximately into two regions. The lateral region contains neurons of relatively small size (30-50 $\mu \mathrm{m}$ in $250-300 \mathrm{gm}$ animals), most of which appear whitish under incident illumination. The medial region contains larger neurons $(70-100 \mu \mathrm{m})$, most of which are yellow-orange. In the medial region, several cells were found to receive marked asymmetrical inputs from sensory stimulation of the lips and tentacles, the input being stronger from the ipsilateral side. The axons of these medial $\mathrm{C}$ cluster cells project ipsilaterally either to the C-PLC or to the C-PC. One such bilateral pair of identifiable neurons, CC5, was found to evoke very widespread activity in neurons in other ganglia.
A

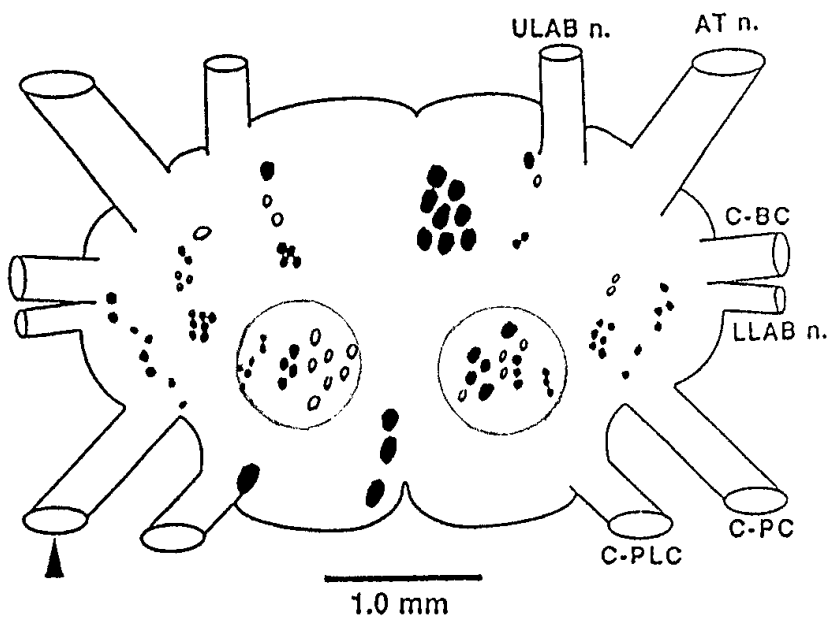

B

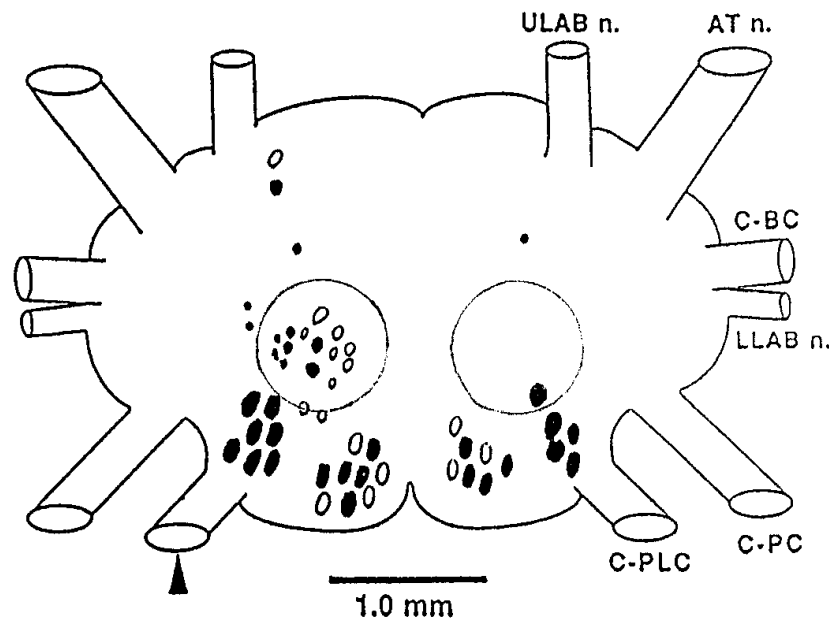

Figure 1. Schematic diagram of the position of cells in the cerebral ganglion that send axons to the pedal $(A)$ or pleural $(B)$ connective. The arrowhead indicates the filled connective. The boundary of the $\mathrm{C}$ cluster is indicated by the circular outline. The figure summarizes the results obtained from five preparations. Typically, one connective was filled with cobalt chloride, and the contralateral connective was filled with nickel chloride so that cells with crossed and uncrossed axons could be distinguished by color (Quicke and Brace, 1979). Cells located toward the dorsal surface are shown as solid; cells toward the ventral surface are shown as open.

\section{Morphology of CC5}

CC5 is located bilaterally in the superficial layer of the caudal medial part of the cerebral C cluster (Fig. 2). It typically appeared to be covered by a layer of cells, but could be revealed by stretching and flattening the ganglion so that the most superficial layer of cells separated partially. The cell is round and yelloworange. Its diameter is $\sim 80 \mu \mathrm{m}$. Intracellular dye fills with Lucifer yellow, and 5(6)-carboxyfluorescein dye revealed that CC5 has a single stout axon that extends into the ipsilateral C-PLC (Fig. 2). Fine branches are given off from the main axon in the neuropil deep to the cells in the A cluster before the axon enters the C-PLC. The main process projects through the C-PLC to the 


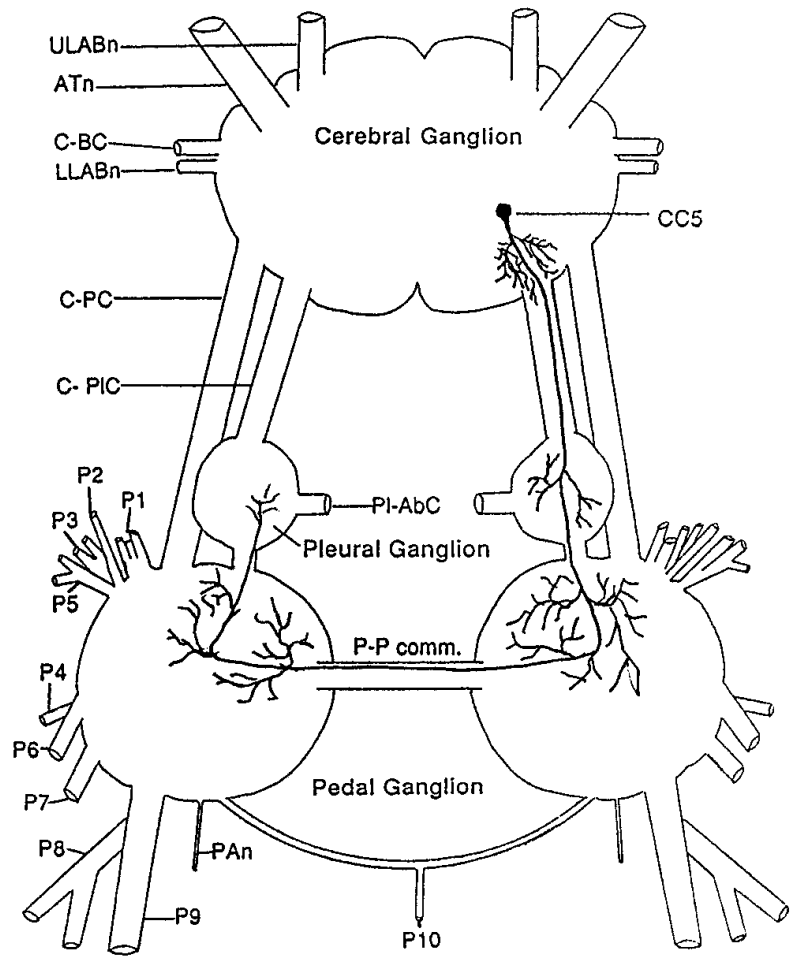

Figure 2. Schematic drawing illustrating the right CC5 neuron and its processes. A symmetrical cell is present in the opposite hemi-ganglion. The drawing is based on visualizations of CC5 neurons in fixed ganglia after fills with Lucifer yellow and on unfixed ganglia after fills with 5(6)-carboxyfluorescein dye $(n=10)$.

ipsilateral pleural-pedal ganglia; the axon then continues through the pedal-pedal commissure to the contralateral pedal-pleural ganglion where it appears to terminate. Many fine branches extend from the main process as it courses through the ipsilateral and contralateral pleural and pedal ganglia.

\section{CC5 receives asymmetric inputs from the lips and tentacles}

On initial penetration, CC5 fired at a high frequency, and then over a period of 10 to $15 \mathrm{~min}$, its firing slowed gradually until it became almost quiescent, exhibiting occasional trains of spontaneous firing or irregular low-frequency spikes. For most experiments, the cell was hyperpolarized slightly to eliminate spontaneous firing. The spike had a relatively rapid rise and fall time and had a half-height width of $6-8 \mathrm{msec}$. The resting potential of the cells was approximately $-50 \mathrm{mV}$, and the spike exhibited an amplitude of $\sim 60 \mathrm{mV}$. A low rate of spontaneous excitatory synaptic inputs was typically observed.

To determine the sensory inputs to CC5, the isolated head preparation was used. Seaweed extract, pieces of seaweed, or mechanical probes were applied to the lips or tentacles. In experiments designed to explore the possible contribution of CC5 to head-turning responses, we initially touched the lips with a piece of seaweed to promote a food-induced arousal state (Kupfermann, 1974a), because head turning to tactile stimuli in intact animals requires that the animal be aroused (Teyke et al., 1990b).

Application of a piece of a tactile stimulus to the lip, anterior tentacle, or rhinophore evoked excitatory responses in CC5 (Fig. $3 A, B)$. Similar responses were evoked by a piece of seaweed. Chemical stimuli lacking a tactile component, provided by seaweed extract, did not produce excitatory effects on CC5. The
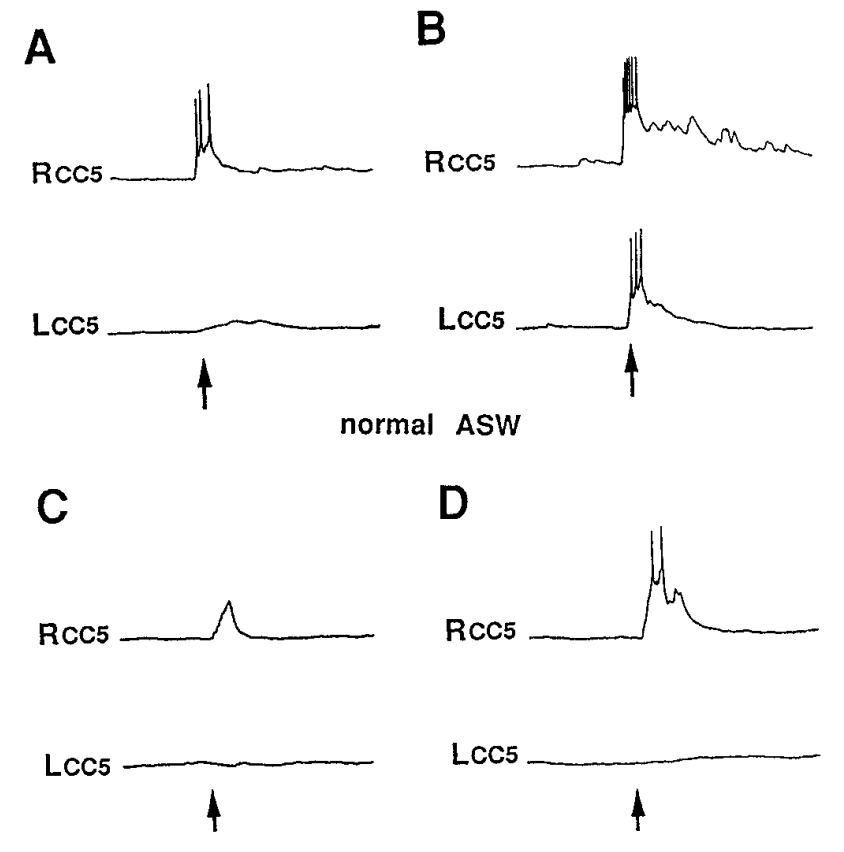

Hi Mg++ Hi Ca++

\section{$\underset{500 \mathrm{~ms}}{ل} 40 \mathrm{mV}$}

Figure 3. Sensory inputs from the tentacles to CC5. $A, B$, Intracellular recordings from the left and right $\mathrm{CC} 5$ in the same preparation were obtained in isolated head preparations. $A$, Contact with a fine von Frey hair applied to one tentacle (the right tentacle) evoked excitatory synaptic input and firing of the ipsilateral CC5 and little input in the contralateral CC5. $B$, When stronger stimulus was pressed strongly to the tentacle, there was excitatory input in both the ipsilateral and contralateral CC5, with the ipsilateral response stronger. $C, D$, In the presence of a solution containing high-divalent cations, synaptic input was seen only in the ipsilateral cell for both weak $(C)$ and strong $(D)$ stimuli $(n=8)$.

evoked responses appeared to exhibit little or no decrement when repeated, in contrast to responses to tactile stimuli, evoked in cerebral B cells (Fredman and Jahan-Parwar, 1977) or in cells involved in siphon, gill, and tail-withdrawal responses (Kupfermann et al., 1970). Although the response did not decrement with repeated stimulation, it exhibited rapid adaptation, and prolonged stimuli were only minimally more effective than brief stimuli. CC5 had a large receptive field, extending from the perioral zone to the most lateral anterior tentacle, and included the rhinophore. Within this receptive field, there was no apparent regional difference in the intensity of the response. This is in contrast to head-turning responses in intact animals in which the responses are stronger with more lateral stimuli (Teyke et al., 1990b).

Weak tactile stimuli (seaweed or a von Frey hair, 0.1-0.3 gm) could evoke synaptic input and spike activity that was primarily or exclusively ipsilateral (Fig. $3 A$ ), whereas stronger stimuli (tip of heat-sealed Pasteur pipette or von Frey hair, 0.5-1.0 gm) evoked responses in the contralateral as well as ipsilateral CC5 (Fig. 3B). However, the contralateral response was much weaker than the ipsilateral response.

When the head ganglia were bathed in a high-divalent cation solution, which suppresses polysynaptic activity, the excitation of CC5 induced by touch to the ipsilateral lip or tentacles still persisted, whereas the excitation of CC5 induced by touch to the contralateral lip or tentacles by either a weak (Fig. $3 C$ ) or strong (Fig. $3 D$ ) stimulus was eliminated or greatly reduced. This sug- 
A1

RPAnHWWHWHHHWHHHH
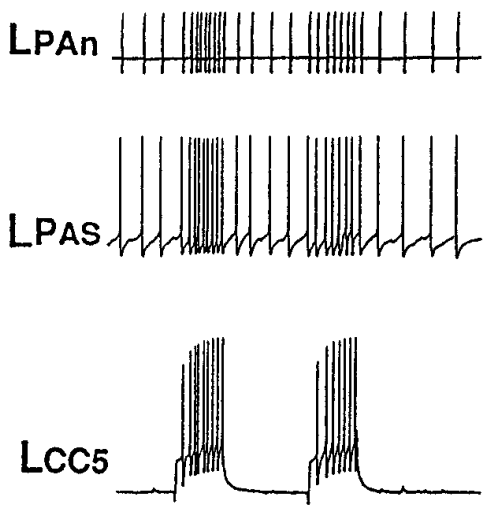

A 2
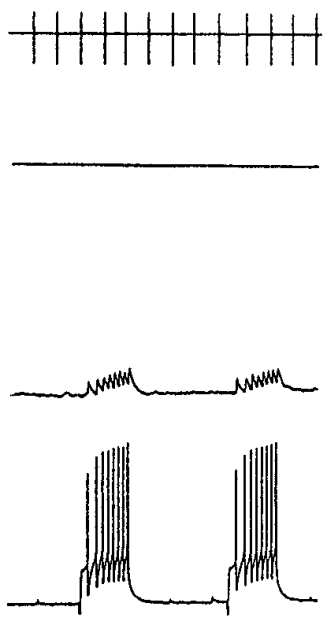

$20 \mathrm{mV}$
$\mathbf{B}$
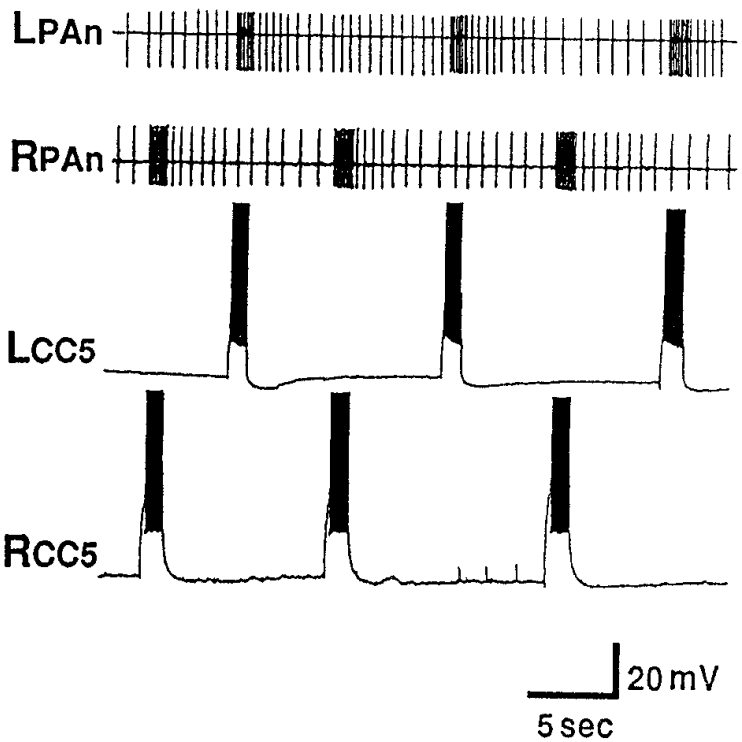

Figure 4. A1, Firing trains of spikes in CC5 excite a neuron with an axon in the ipsilateral PAn but have no effect on the neuron in the contralateral PAn. $A 2$, When the ipsilateral PAS neuron was hyperpolarized, CC5 failed to evoke spikes in the cell, and the extracellularly recorded spike of PAS was eliminated $(n=6) . B$, Simultaneous extracellular recordings from the LPAn and RPAn and intracellular recordings from the left and right CC5 neurons show the strictly unilateral actions of CC5 on the PAS $(n=10)$.

gests that the excitation $\mathrm{CC} 5$ receives from the ipsilateral field is mediated at least in part by monosynaptic connections, whereas the contralateral input may be mediated primarily by interneurons, although we cannot exclude completely the possibility that the high-divalent cation solution produced conduction block of the contralateral afferent rather than block of interneurons.

\section{Synaptic output of CC5 to pedal arterial shortener motor neurons}

To initially study the possible role of CC5 in head turning and other behaviors, we fired the neuron and recorded from different nerves. We found that firing of CC5 altered the activity of units recorded from a variety of nerves. A particularly striking effect was observed in the PA nerve, in which the activity of a single unit was excited strongly by firing CC5 (Fig. 4). Firing of CC5 resulted in increased activity of the ipsilateral PA nerve, but had no effect on the contralateral PA nerve (Fig. 4B).

Previously, Skelton and Koester (Skelton and Koester, 1992) reported that the LPAn and RPAn contain the axon of a motor neuron, called the pedal artery shortener (or PAS) for which firing causes contraction of the PA. They demonstrated that each PAS neuron provides the major and perhaps sole excitation to the ipsilateral PA. We therefore recorded next from the PAS neuron (located in the pedal ganglion) and found that this neuron receives a strong excitatory synaptic connection from $\mathrm{CC} 5$, and the EPSP was capable of driving PAS at a relatively high rate (Fig. 4A1). When PAS was hyperpolarized, CC5 no longer evoked spikes in PAS, and no spike activity appeared in the PAn (Fig. $4 A 2$ ). Each spike of CC5 produced a constant latency EPSP in the ipsilateral PAS (Fig. 5A1). Our evidence suggests that the EPSP is a monosynaptic chemical synaptic potential. The EPSP was blocked or reduced in a solution containing low calcium and high magnesium (Fig. 5A2) and persisted in a high-divalent cation solution (Fig. 5B2).
Additional studies indicated that CC5 may provide the only excitatory input from the cerebral ganglion to PAS via the C-PLC. Extracellular stimulation of the C-PLC with a brief electrical pulse evoked an EPSP in PAS. In ASW, pulses of progressively greater intensity evoked progressively larger EPSPs (Fig. $6 A$ ). The EPSPs

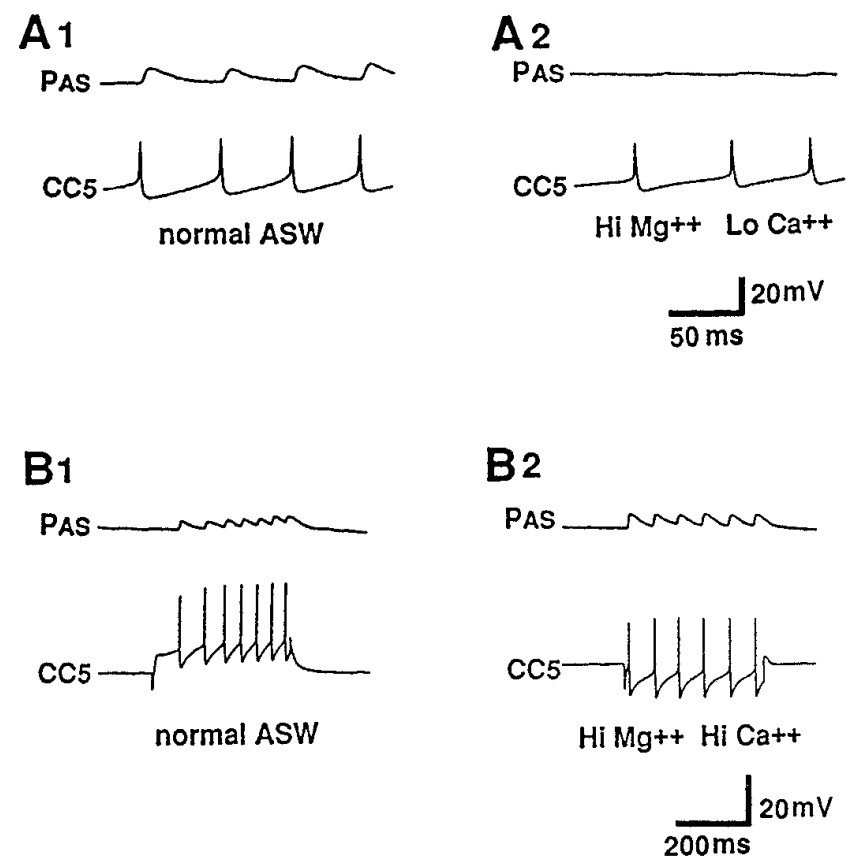

Figure 5. A1, CC5 evokes an EPSP in the ipsilateral PAS motor neuron. $A 2$, The EPSP appears to be mediated chemically and is reduced or blocked in a solution containing increased $\mathrm{Mg}^{2+}$ and decreased $\mathrm{Ca}^{2+} . \mathrm{B1}$, $B 2$, The EPSP persists when the preparation is bathed in a solution containing high-divalent cations $(n=6)$. 


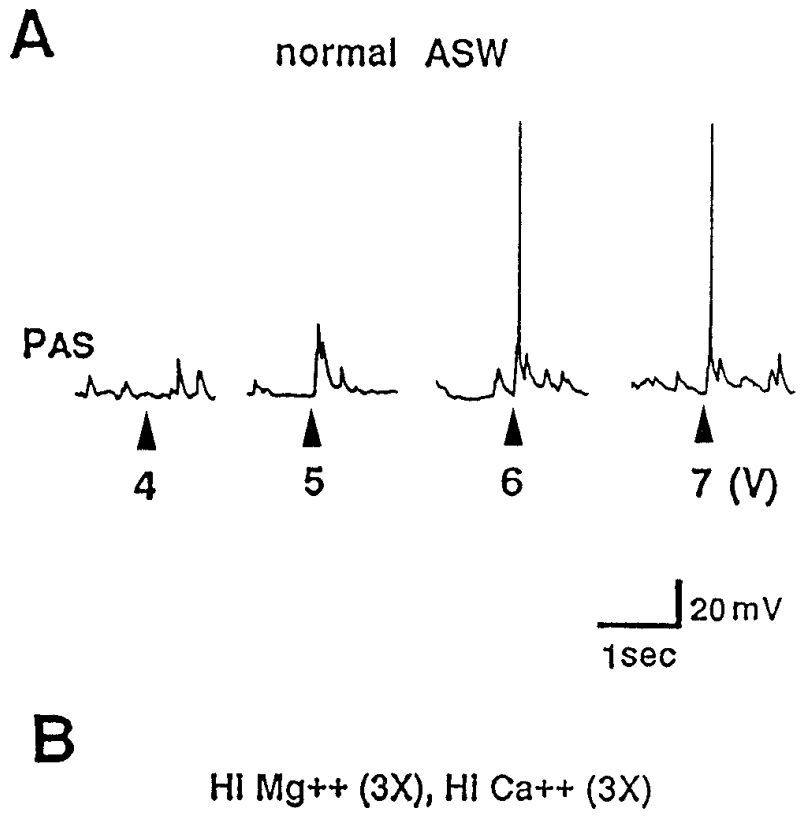

PAS

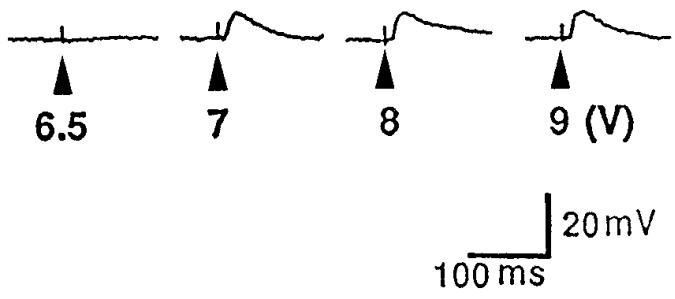

Figure 6. Electrical stimulation of the pleural end of a severed C-PLC evokes an EPSP in PAS. Single shocks of the pleural end of the C-PLC were presented at gradually increasing intensities (voltage indicated below traces). $A$, In normal ASW, PAS exhibits small spontaneous EPSPs, probably of origin from the pedal or pleural ganglia. In this experiment, a $5 \mathrm{~V}$ shock evoked an EPSP with multiple components. Higher voltages brought in a larger EPSP that appeared to be polysynaptic. $B$, In highdivalent ASW, a smooth EPSP was evoked in PAS at a relatively sharp threshold, and no additional increase in size of the EPSP occurred with shocks of increasing intensity $(n=5)$.

contained multiple peaks that occurred at variable latencies, suggesting that the EPSPs were caused, at least in part, by polysynaptic activity evoked in the pedal or pleural ganglia. In a high-divalent cation solution that reduces polysynaptic activity, stimulation of the C-PLC evoked a simple EPSP that occurred in an all-or-none manner (Fig. 6B). The size of the EPSP was unchanged when larger pulses were used. With very large pulses, multiple EPSPs sometimes were evoked, and these appeared to be caused by multiple firing of a single axon in the connective. We also found that extracellular stimulation of the C-PC did not evoke an obvious EPSP in PAS. In fact, stimulating the C-PC evoked inhibitory inputs to PAS (Fig. 7). The presence of the IPSP does not exclude the existence of a simultaneous EPSP. Elsewhere, we show that the inhibitory input comes from, at least in part, an inhibitory connection of identified cerebral neuron C-PR (Xin et al., 1996).

CC5 is necessary and sufficient for the mediation of the unilateral PA-shortening component of a local withdrawal reflex

Given that CC5 provides excitatory input to PAS, we examined whether firing of CC5 was capable of firing PAS sufficiently to

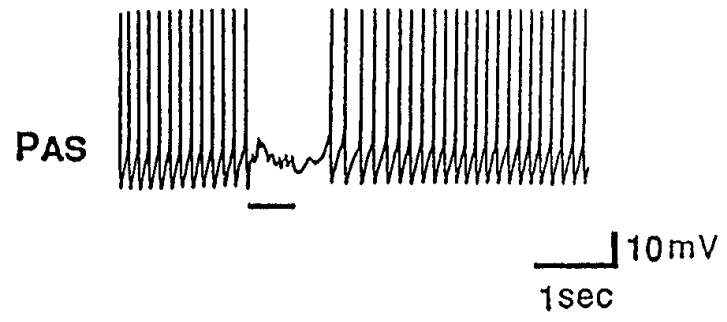

Figure 7. A train of electrical stimuli (line under trace) applied to the pedal end of a severed C-PC evokes IPSPs in the PAS motor neuron $(n=5)$.

evoke contractions of the PA. We found that firing of CC5 produced an arterial-shortening response for which the magnitude was proportional to the rate of firing of $\mathrm{CC} 5$, within a range of $\sim 3-15$ spikes/sec (Fig. $8 A$ ). This range of firing is similar to that evoked by brief tactile stimuli applied to the tentacle (see Figs. 3, 9). The shortening of the PA also was dependent on the firing frequency of the pedal artery motor neuron PAS (Fig. $8 B$ ). The contraction was related linearly to PAS frequency over a range of $5-15 \mathrm{~Hz}$ (Fig. $8 C$ ). The shortening produced by $\mathrm{CC} 5$ appeared to be accounted for completely by the evoked firing of the PAS, because the contraction evoked by CC5 was blocked completely when the firing of the PAS was prevented by means of hyperpolarizing current (Fig. 8D).

Because a weak tactile stimulus effectively fires only one CC5 neuron, and one CC5 neuron is capable of evoking a contraction of the PA, we examined next whether an individual CC5 was necessary for the control of an arterial-shortening response that might be evoked by a weak tactile stimulus applied to a tentacle. In preparations consisting of the head ganglia with the lips, anterior tentacles, and PA attached, we found that a weak tactile stimulus applied to a tentacle consistently evoked a local contraction of the tentacle, accompanied by a shortening of the ipsilateral PA together with firing of CC5 (Fig. 9A,B1,B3) and excitatory input to PAS (Fig. 10A). The firing of CC5 was necessary for the arterial-shortening response, because the arterial contraction (Fig. 9B2) and excitatory input to PAS (Fig. 10C) evoked by the tactile stimuli was blocked completely when CC5 was hyperpolarized. CC5 also appeared to be sufficient to evoke the arterial shortening, because direct firing of $\mathrm{CC} 5$ at a rate approximately comparable to that produced by brief tactile stimuli produced arterial shortening (Fig. 9B4) and excitatory input to PAS (Fig. $10 B$ ) that was approximately comparable to that evoked by the tactile stimuli. Even with a relatively strong tactile stimulus, CC5 appeared to be necessary (Fig. 11C) and sufficient (Fig. 11B) for the firing of the PAS neuron. In contrast to arterial shortening, CC5 appeared to be neither necessary nor sufficient for the tentacle withdrawal component of the local withdrawal response. Visual observation of the tentacles revealed that firing of CC5 produced little or no tentacle withdrawal, and a robust tentacle withdrawal still occurred when CC5 was hyperpolarized.

Data obtained from recordings from the PAn artery nerve in free-moving animals were consistent with the idea that CC5 may be necessary and sufficient for the arterial-shortening component of a local withdrawal response. Weak tactile stimuli applied to a tentacle evoked a brief discharge in the PA nerve (Fig. 12A). When the C-PLC was severed and the animal permitted to recover, tactile stimuli applied to the head no longer affected activity in the PA nerve (Fig. 12 B). This lesion eliminates the only direct connection that CC5 provides to the ipsilateral PAS neuron. 


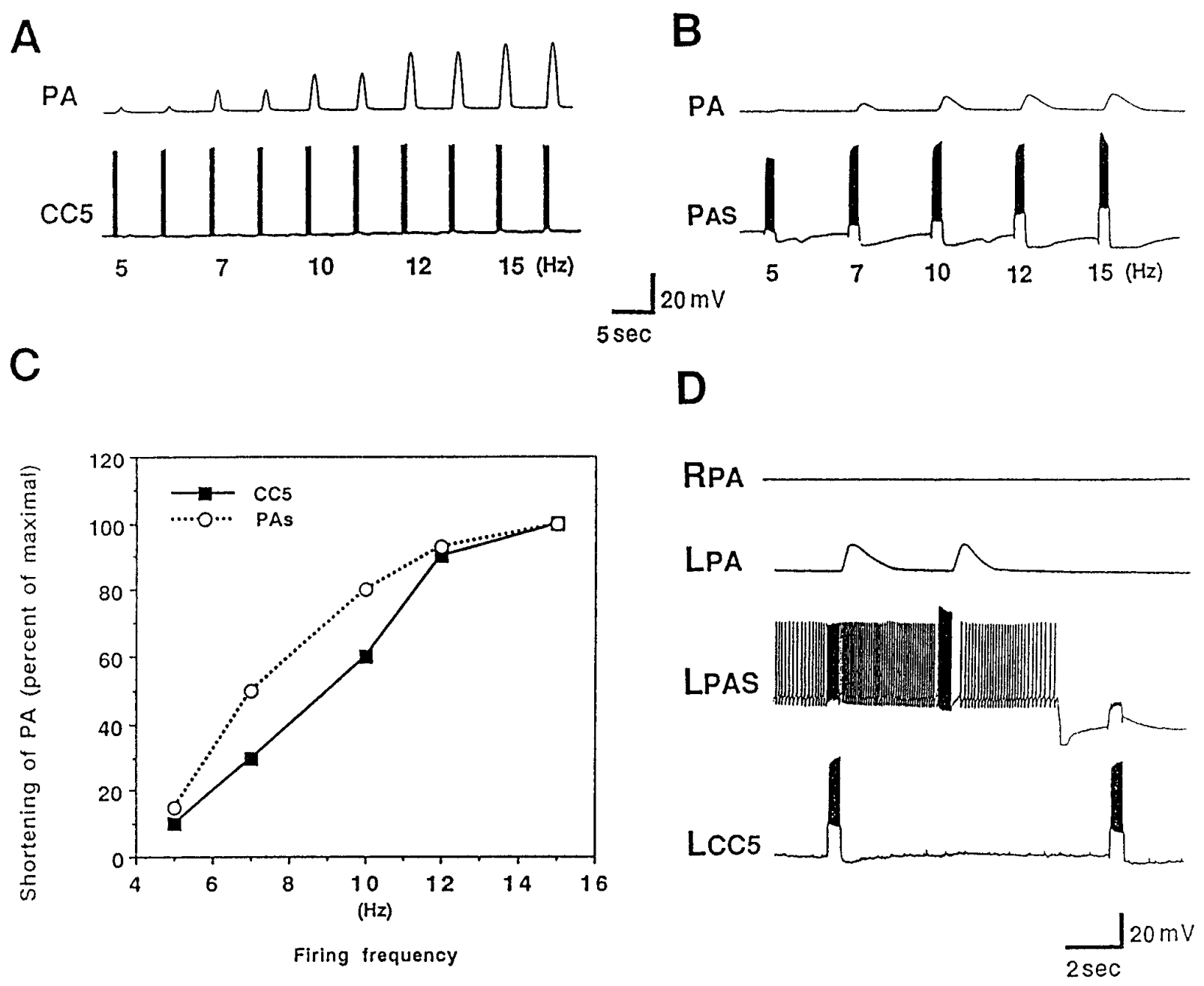

Figure 8. Firing of PAS and CC5 evokes contractions of the PA. A, Contraction of the PA as a function of different firing frequencies of CC5. CC5 was fired by means of $1 \mathrm{sec}$ depolarizing pulses with progressively increasing current. The frequency of the resulting spikes is indicated below the CC5 traces. Contraction of the artery was recorded by an isotonic displacement transducer $(n=7)$. B, Magnitude of shortening of the PA as a function of the frequency of firing of PAS during bursts of 2 sec duration $(n=7)$. $C$, Relationship of spike frequency of CC5 (squares) or PAS (circles) to the magnitude of shortening of the PA. D, Evidence that PA shortening evoked by CC5 is mediated entirely by the ipsilateral PAS neuron. Initially, the left CC5 was fired by a long depolarizing pulse, and the left PAS was at resting potential. PAS fired because of the excitation from CC5, and the left PA contracted. When PAS was hyperpolarized so that CC5 did not evoke any spikes in PAS (second burst of spikes in CC5), the arterial contraction was blocked completely. Between the two CC5 stimulations, PAS was fired by a long depolarizing pulse so that it fired similarly to that evoked by firing of CC5. Direct firing of PAS evoked a contraction comparable to that evoked by CC5 $(n=5)$.

Although in itself this experiment is not definitive, because numerous axons other than CC5 are present in the connective, the data support the findings presented in Figures 8 and 9 that indicate that lack of the response to tactile stimuli was attributable to the disconnection of CC5 to the PAS neuron.

\section{DISCUSSION}

The original goal of these experiments was to identify neurons that might be involved in mediating neck movements and headturning behavior. Based on the criteria of receiving asymmetrical inputs and having asymmetrical outputs, we identified a candidate neuron, $\mathrm{CC} 5$, that is located in the $\mathrm{C}$ cluster region of the cerebral ganglion. In this paper, we report that each CC5 neuron provides a powerful monosynaptic excitatory input to the ipsilateral PAS neuron, a bilaterally symmetrical identified motor neuron that controls contractions of the PA (Skelton and Koester, 1992). Shortening of this artery is a concomitant of behaviors in which the neck of the animal contracts. The arterial shortening may serve to take up slack in the artery (Skelton and Koester, 1992) or perhaps may provide some motive force that aids in neck and head movements. The evidence presented in the present paper suggests that CC5 is involved in a component of the constellation of responses associated with shortening of the neck. In the present paper, we have studied the role of CC5 in mediating an arterialshortening response that occurs as a component of tentacle and head-withdrawal responses to tactile stimuli applied to the anterior tentacles. Two types of behaviors can be distinguished: a local tentacle withdrawal in response to a weak stimulus, and head withdrawal, which occurs when a strong or noxious stimulus is applied (Teyke et al., 1989). Both responses involve a concomitant shortening of the PA. In a companion paper (Xin et al., 1996), we present evidence for a role of CC5 in controlling muscles of the neck that appear to be involved in head turning, withdrawal, and other behaviors that involve movements of the head and neck.

The data obtained in this study indicate that the ipsilateral CC5 


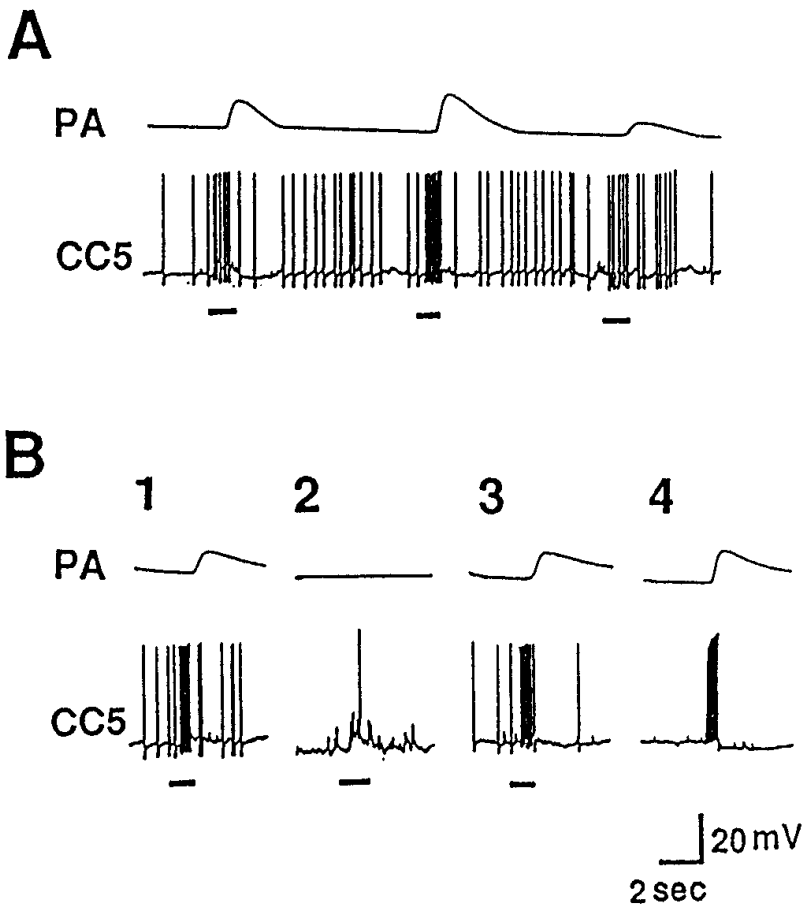

Figure 9. Role of CC5 in the arterial-shortening component of a local withdrawal response. $A$, In a semi-intact preparation, touches (line under trace) to the tentacle with a piece of seaweed evoked firing of CC5 and contraction of the ipsilateral PA. For the last touch in the series, CC5 fired at a relatively low frequency, and only a small contraction was evoked. $B$, Effect of hyperpolarizing CC5 on the arterial contraction reflex. The tentacle was touched three times $(B 1-B 3)$, and CC5 also was stimulated directly (B4). B1, When CC5 was at rest potential, a tactile stimulus evoked spikes in $\mathrm{CC} 5$, and an arterial contraction occurred. B2, When CC5 was hyperpolarized, the touch with seaweed evoked only a single spike in CC5, and there was no contraction of the artery. B3, Direct firing of CC5 at a rate comparable to that evoked by the tactile stimulus evoked a comparable contraction of the artery $(n=6)$.

neuron is necessary and sufficient for the brisk unilateral arterialshortening response that is evoked by a relatively weak ipsilateral tentacular tactile stimulus. This conclusion arises from converging lines of evidence: (1) The CC5 neurons are excited by tactile stimuli applied to the head, and weak tactile stimuli only fire the ipsilateral CC5 neuron at a rate sufficient to drive a contraction. (2) Each CC5 neuron makes powerful monosynaptic connections to the ipsilateral PAS motorneuron, and firing of the CC5 cells at rates comparable to those produced by sensory inputs can evoke arterial contractions that also are comparable to those evoked by sensory stimulation. (3) Arterial shortening evoked by weak tactile stimuli is eliminated completely when the ipsilateral CC5 is removed from the circuit by hyperpolarization. The results obtained from recordings of the excitatory follower cell of CC5, PAS, before and after lesioning of the pleural connective are consistent with the interpretation that arterial shortening is mediated by $\mathrm{CC} 5$. We have not determined the degree to which CC5 contributes to the other components that make up the local withdrawal response, but it clearly is not necessary for the tentacular movements that are evoked by the same stimulus. These movements can be evoked by firing of cerebral B cells (Fredman and Jahan-Parwar, 1977; Rosen et al., 1979), neurons that are not excited by CC5. Furthermore, small tentacular movements can be elicited by tactile stimuli even in the absence of the CNS (Y. Xin, K. R. Weiss, and I. Kupfermann, unpublished observations), presumably mediated by a peripheral nerve net.
Narrowly construed, CC5 appears to be a rare bona fide example of a command neuron (Kupfermann and Weiss, 1978, 1986) for the arterial-shortening component of the total withdrawal reaction. The notion of defining a command neuron as a cell that is necessary and sufficient for a specific behavior has been criticized from a number of points of view [Kupfermann and Weiss, 1978, 1986 (and references therein)], but nevertheless the idea that certain neurons can exert extraordinarily powerful effects on behavior remains attractive, and several examples of such neurons have been described in both invertebrates and vertebrates (Nolen and Hoy, 1984; Didomenico et al., 1988; Frost and Katz, 1996). There are a number of potential problems encountered specifically by classifying $\mathrm{CC} 5$ as a command neuron, although, operationally, CC5 meets the criteria of necessity and sufficiency for a particular response. First, it might be argued that CC5 is odd in that it functions as a command neuron only for weak stimuli. Any reflex response will have a threshold level at which it is evoked and at that threshold, any neuron that is part of the circuit will be necessary for the response (although it need not be sufficient). Typically, however, the arterial-shortening response evoked by a weak stimulus or by CC5 firing is well above the noise and decidedly stronger than the minimal contraction that can be evoked. Furthermore, despite extensive search, we have not obtained any evidence that CC5 is one of a group of similar interneurons, any one of which could reach threshold and produce a small response. A second potential problem with considering CC5 a command neuron is that the available evidence indicates that it is necessary and sufficient for only a component of a larger response, so that the command function of the neuron could be considered trivial. To some extent, however, virtually all behaviors can be considered components of larger behavioral units. It is ultimately a matter of theoretical preference whether it is useful or illuminating to extend the notion of command to include cells that elicit components of a more general response. The Mauthner cell of fish provides an example of an individual identified vertebrate neuron that appears to control a small but functionally important component of a complex behavior (Didomenico et al., 1988).

The current work emphasizes the need to categorize command neurons in terms of specific behaviors that are defined in terms of movements together with the eliciting stimuli (Kupfermann and Weiss, 1978), because the same movement can be elicited by different stimuli, and slightly different stimuli may elicit markedly different responses. The neural controls of a specific response very well could be different for each class of stimuli eliciting the response. Thus, tentacle movement can be elicited by a weak stimulus as well as by a strong stimulus. In fact, when a strong tactile stimulus is applied to the head, instead of a local withdrawal response, a defensive withdrawal of the whole head is evoked. A strong stimulus activates both CC5 cells, and even for a strong stimulus, the excitation of a PA is virtually eliminated by hyperpolarizing the ipsilateral CC5. Thus, it is likely that acting conjointly, the cells mediate bilateral arterial shortening, i.e., they act as a two-cell "command system." Head withdrawal, however, involves inputs to Bn cells (Teyke et al., 1989) and probably other neurons that mediate the major contraction movements of the neck. Our data provide no evidence that the CC5 cells in themselves can evoke activity in the Bn cells and, in fact, they may weakly inhibit Bn cells. The CC5 cells, however, may contribute to neck movements by means of their excitatory connections to motor neurons in the pedal ganglia (our unpublished observations). 
A

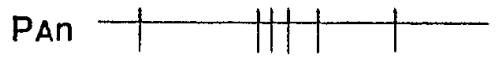

PAS UUU

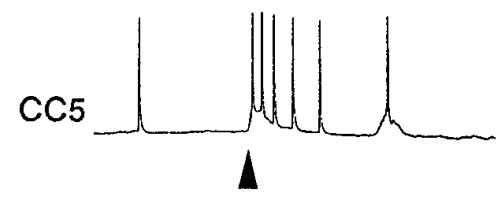

B
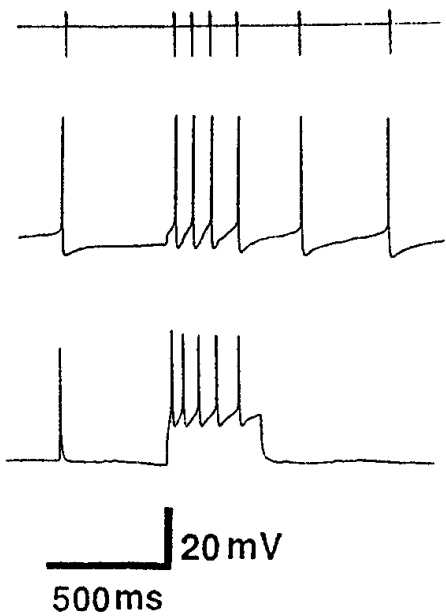

C
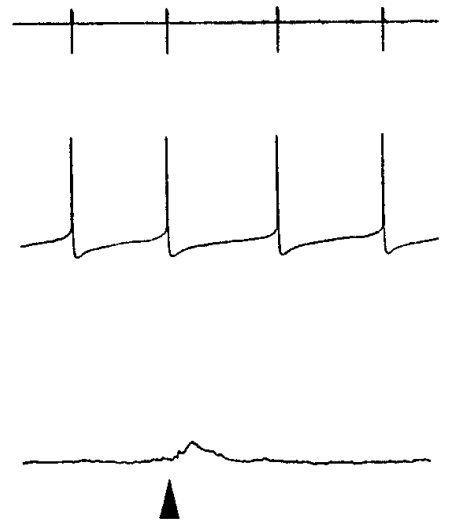

Figure 10. CC5 mediates excitatory input to PAS evoked by tactile stimulation of a tentacle. The activity of the ipsilateral PAS recorded intracellularly together with an extracellular recording from the PAn. $A$, A weak tactile stimulus applied to the ipsilateral tentacle evoked excitatory input to CC5, followed by excitatory input to PAS. $B$, When CC5 was fired directly by a depolarizing pulse that was adjusted to evoke firing similar to that evoked by the tactile stimulus, PAS fired approximately the same as it did after the tactile stimulus (compare with $A$ ). $C$, When CC5 was hyperpolarized, the tactile stimulus failed to evoke spikes in CC5, and the excitatory input to PAS was eliminated $(n=6)$.

Discussion of what terms to use to describe a neuron may strike some investigators as a useless exercise of hair splitting. Others may find that such classification helps organize possibly confusing functional analyses and adds an intuitive dimension that can supplement more abstract computational or mathematical ac- counts of the functional operation of circuits. An important recent notion related to that of command neuron, but lacking the behavioral and philosophical implications of command, is that of the "influential" neuron (Arshavsky et al., 1988). Although originally defined as a neuron that has some effect on a rhythmic motor
A

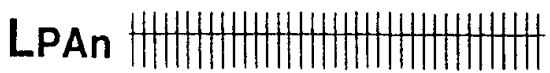

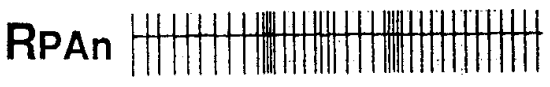

LCC5
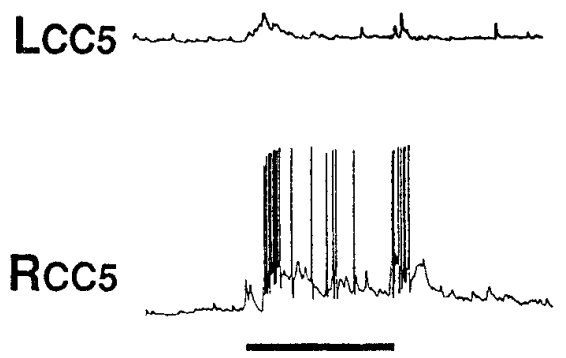

B
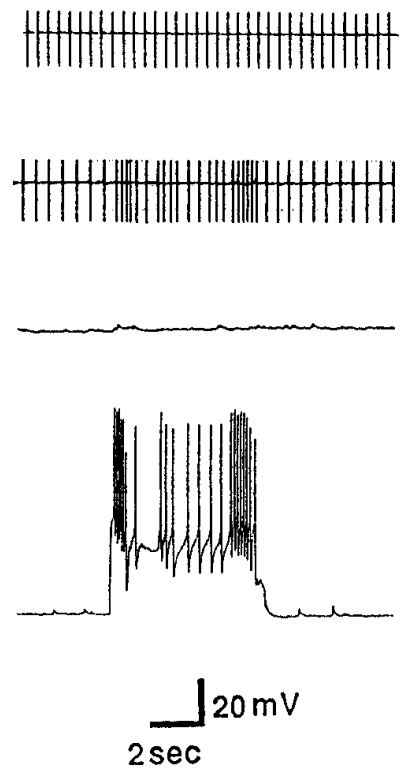

2 sec
C
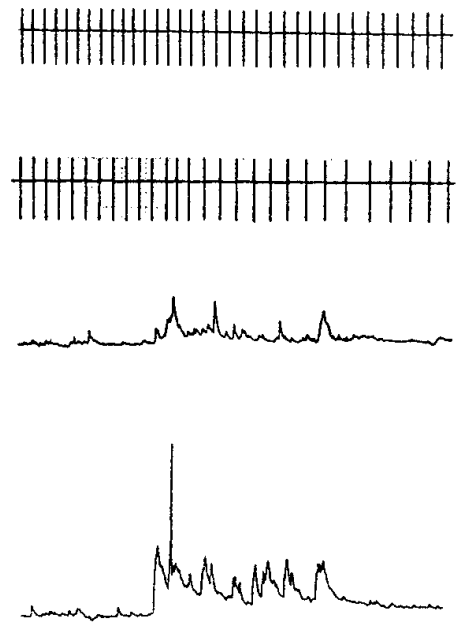

Figure 11. The firing of PAS after relatively strong tactile stimuli appears to be mediated by CC5. In this experiment, both the left and right CC5 cells were impaled. $A$, The two CC5 cells were slightly hyperpolarized $(\sim 5 \mathrm{mV})$ in order to decrease spontaneous activity. A relatively strong and prolonged stimulus (indicated by the bar under the trace) applied to the right tentacle produced strong excitatory input to the ipsilateral CC5 and firing of the PAS motor neuron as monitored by its spike recorded extracellularly in the RPAn. The stimulus evoked a weaker input to the contralateral CC5 that failed to fire the hyperpolarized cell and consequently did not alter the activity of the left PA motor neuron recorded in the LPAn. Termination of the stimulus evoked what appeared to be an off response. $B$, Direct firing of the right CC5 in a pattern similar to that produced by the tactile stimulus also evoked firing of the ipsilateral PAS neuron monitored in RPAn. $C$, The increased firing of PAS after the tactile stimulus was eliminated completely by hyperpolarizing the CC5s another $15 \mathrm{mV}$ so that their spiking was largely eliminated $(n=6)$. 
A

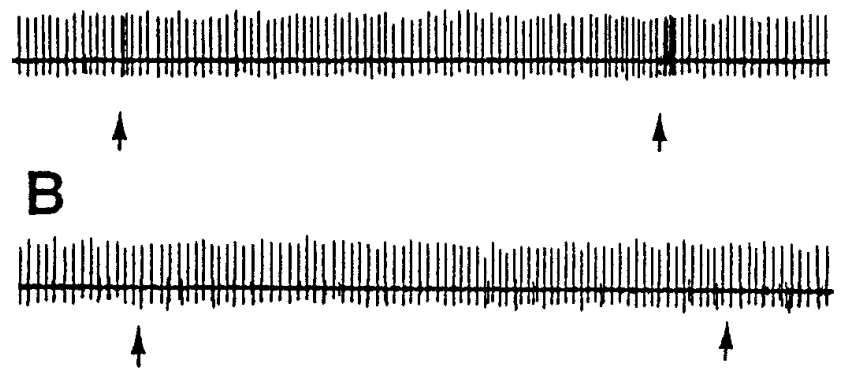

4 Seaweed Touch

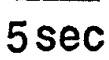

Figure 12. Activity of PAS recorded extracellularly from the PAn in a free-moving animal. $A$, A weak touch to the ipsilateral anterior tentacle by means of a piece of seaweed (similar results were obtained by a pure tactile stimulus) evoked a brief relatively high-frequency discharge in the nerve. $B$, After sectioning of the C-PLC and recovery from surgery, touch no longer evoked any responses in the nerve $(n=8)$.

output (independent of its functional role), the concept can be extended to include neurons meeting one of two criteria: (1) the neuron exerts strong effects on one or more specific neurons, or (2) the neuron has relatively weak effects, but its effects are widespread, that is, its firing excites or inhibits large number of neurons. The evidence presented in this paper indicates that CC5 is an influential neuron in the first sense, because it appears to provide a major source of excitatory input to a motor neuron (PAS), which itself provides the major or only source of control of a particular muscle and behavioral response. In a companion paper (Xin et al., 1996), we provide evidence that CC5 also is an influential neuron in the second sense, in that firing of CC5 has effects on numerous other neurons, and it appears to contribute to components of behaviors during a wide variety of rhythmic as well as nonrhythmic responses, including withdrawal, locomotion, head turning, head lifting, and feeding.

\section{REFERENCES}

Alevizos A, Weiss KR, Koester J (1991) Synaptic actions of identified peptidergic neuron R15 in Aplysia. I. Activation of respiratory pumping. J Neurosci 11:1263-1274.

Arch S, Smock T (1977) Egg-laying behavior in Aplysia californica. Behav Biol 19:45-54.

Arshavsky YI, Deliagina TG, Orlovsky GN, Panchin YV (1988) Control of feeding movements in the freshwater snail Planorbis corneus. III. Organization of the feeding rhythm generator. Exp Brain Res 70:332-341.

Bernheim SM, Mayeri E (1995) Complex behavior induced by egg-laying hormone in Aplysia. J Comp Physiol [A] 176:131-136.

Celebrini S, Newsome WT (1994) Neuronal and psychophysical sensitivity to motion signals in extrastriate area MST of the macaque monkey. J Neurosci 14:4109-4124.

Churchland PS, Sejnowski TJ (1992) The computational brain. Cambridge: MIT.

Delaney K, Gelperin A (1990) Cerebral interneurons controlling fictive feeding in Limax maximus. II. Initiation and modulation of fictive feeding. J Comp Physiol [A] 166:311-326.

Didomenico R, Eaton RC (1988) Seven principles for command and neural causation of behavior. Brain Behav Evol 31:125-140.

Didomenico R, Nissanov J, Eaton RC (1988) Lateralization and adaptation of a continuously variable behavior following lesions of a reticulospinal command neuron. Brain Res. 473:15-28.
Ferguson GP, Ter Maat A, Parsons DW, Pinsker HM (1989) Egg laying in Aplysia. I. Behavioral patterns and muscle activity of freely behaving animals after selectively elicited bag cell discharges. J Comp Physiol [A] $164: 835-847$

Fetz EE (1992) Are movement parameters recognizably coded in the activity of single neurons? Behav Brain Sci 15:679-690.

Fredman SM, Jahan-Parwar B (1977) Identifiable cerebral motoneurons mediating an anterior tentacular withdrawal reflex in Aplysia. J Neurophysiol 40:608-615.

Fredman SM, Jahan-Parwar B (1983) Command neurons for locomotion in Aplysia. J Neurophysiol 49:1092-1117.

Frost WN, Katz PS (1996) Single neuron control over a complex motor program. Proc Natl Acad Sci USA 93:422-426.

Gardner D (1971) Bilateral symmetry and internal organization in the buccal ganglia of Aplysia. Science 173:550-553.

Getting PA (1989) Emerging principles governing the operation of neural networks. Annu Rev Neurosci 12:185-204.

Hooper SL, Moulins M (1989) Switching of a neuron from one network to another by sensory-induced changes in membrane properties. Science 244:1587-1589.

Jahan-Parwar B, Fredman SM (1976) Cerebral ganglion of Aplysia: cellular organization and origin of nerves. Comp Biochem Physiol [A] 54:347-357.

Kuenzi FM, Carew TJ (1994) Head waving in Aplysia californica. III. Interganglionic pathways underlying the coordination and control of searching movements. J Exp Biol 195:75-90.

Kupfermann I (1974a) Feeding behavior in Aplysia: a simple system for the study of motivation. Behav Biol 10:1-26.

Kupfermann I (1974b) Dissociation of the appetitive and consummatory phases of feeding behavior in Aplysia: a lesion study. Behav Biol 10:89-97.

Kupfermann I, Weiss KR (1978) The command neuron concept. Behav Brain Sci 1:3-39.

Kupfermann I, Weiss KR (1986) Command performance. Behav Brain Sci 9:736-739.

Kupfermann I, Castellucci V, Pinsker H, Kandel E (1970) Neuronal correlates of habituation and dishabituation of the gill-withdrawal reflex in Aplysia. Science 167:1743-1745.

Leonard JL, Lukowiak K (1986) The behavior of Aplysia californica Cooper (Gastropoda, Opisthobranchia). I. Ethogram. Behaviour 98:320-360.

Lockery SR, Kristan Jr WB (1990) Distributed processing of sensory information in the leech. II. Identification of interneurons contributing to the local bending reflex. J Neurosci 10:1816-1829.

McCrohan CR, Kyriakides MA (1989) Cerebral interneurones controlling feeding motor output in the snail Lymnaea stagnalis. J Exp Biol 147:361-374.

Meyrand P, Simmers J, Moulins M (1994) Dynamic construction of a neural network from multiple pattern generators in the lobster stomatogastric nervous system. J Neurosci 14:630-644.

Nagahama T, Weiss KR, Kupfermann I (1993) Effects of cerebral neuron C-PR on body postural muscles associated with a food-induced arousal state in Aplysia. J Neurophysiol 70:1231-1243.

Nolen TG, Hoy RR (1984) Initiation of behavior by single neurons: the role of behavioral context. Science 226:992-993.

Preston RJ, Lee RM (1973) Feeding behavior in Aplysia californica: role of chemical and tactile stimuli. J Comp Physiol Psychol 82:368-381.

Quicke DLJ, Brace RC (1979) Differential staining of cobalt- and nickelfilled neurones using rebeanic acid. J Microsc 115:161-163.

Rao G, Barnes CA, McNaughton BL (1986) Intracellular fluorescent staining with carboxyfluorescein: a rapid and reliable method for quantifying dye-coupling in mammalian central nervous system. J Neurosci Methods 16:251-263.

Rosen SC, Weiss KR, Kupfermann I (1979) Response properties and synaptic connections of mechanoafferent neurons in cerebral ganglion of Aplysia. J Neurophysiol 42:954-974.

Rosen SC, Teyke T, Miller MW, Weiss KR, Kupfermann I (1991) Identification and characterization of cerebral-to-buccal interneurons implicated in the control of motor programs associated with feeding in Aplysia. J Neurosci 11:3630-3655.

Skelton ME, Koester J (1992) The morphology, innervation and neural control of the anterior arterial system of Aplysia californica. J Comp Physiol [A] 171:141-155.

Steinberg TH, Newman AS, Swanson JA, Silverstein SC (1987) Macrophages possess probenecid-inhibitable organic anion transporters that 
remove fluorescent dyes from the cytoplasmic matrix. J Cell Biol 105:2695-2702.

Stewart WW (1978) Functional connections between cells as revealed by dye-coupling with a highly fluorescent napththalimide tracer. Cell $14: 741-759$.

Susswein AJ, Weiss KR, Kupfermann I (1978) The effects of food arousal on the latency of biting in Aplysia. J Comp Physiol [A] 123:31-41.

Susswein AJ, Gev S, Achituv Y, Markovich S (1984) Behavioral patterns of Aplysia fasciata along the mediterranean coast of Israel. Behav Neural Biol 41:7-22.

Teyke T, Weiss KR, Kupfermann I (1989) A subpopulation of cerebral B cluster neurones of Aplysia californica is involved in defensive head withdrawal but not appetitive head movements. J Exp Biol 147:1-20.

Teyke T, Weiss KR, Kupfermann I (1990a) An identified neuron (CPR) evokes neuronal responses reflecting food arousal in Aplysia. Science 247:85-87.
Teyke T, Weiss KR, Kupfermann I (1990b) Appetitive feeding behavior of Aplysia: behavioral and neural analysis of directed head turning. J Neurosci 10:3922-3934.

Teyke T, Weiss KR, Kupfermann I (1992) Orientation of Aplysia californica to distant food sources. J Comp Physiol [A] 170:281-289.

Walters ET (1986) Directional control and the functional organization of defensive responses in Aplysia. J Comp Physiol [A] 159:339-351.

Wu J-Y, Cohen LB, Falk CX (1994) Neuronal activity during different behaviors in Aplysia: a distributed organization? Science 263:820-823.

Xin Y, Kupfermann I (1995) Neuronal pathways mediating head turning behavior in Aplysia. Neurosci Lett 186:197-199.

Xin Y, Weiss KR, Kupfermann I (1996) An identified interneuron that contributes to aspects of six different behaviors in Aplysia. J Neurosci, in press.

Young MP, Yamane S (1992) Sparse population coding of faces in the inferotemporal cortex. Science 256:1327-1331. 